\title{
Virtual Reality: Digital or Fictional?
}

Neil McDonnell

University of Glasgow

Nathan Wildman

Tilburg University

\begin{abstract}
Are the objects and events that take place in Virtual Reality genuinely real? Those who answer this question in the affirmative are realists, and those who answer in the negative are irrealists. In this paper we argue against the realist position, as given by Chalmers (2017), and present our own preferred irrealist account of the virtual. We start by disambiguating two potential versions of the realist position - weak and strongand then go on to argue that neither is plausible. We then introduce a Waltonian variety of fictionalism about the virtual, arguing that this sort of irrealist approach avoids the problems of the realist positions, fits with a unifying theory of representational works, and offers a better account of the phenomenology of engaging in virtual experiences.
\end{abstract}

\section{Keywords}

Virtual reality, realism, irrealism, fictionalism, Ken Walton.

In Longbow, a virtual reality application for the HTC Vive, you defend your castle from an advancing horde of cartoonish grey raiders by, as the title suggests, filling them full of arrows. It features several apparent objects - the castle walls you stand upon, the longbow in your hands, the arrows you fire, and, of course, the comical grey army. Further, playing Longbow involves the apparent occurrence of several events: for example, your scanning the valley before your castle wall, your drawing the bowstring, and, eventually, the destruction of your doomed castle gate. But what is the ontological sta- 
tus of these objects and these events - are they, in some sense, real?

Virtual realists say that virtual objects (e.g. my virtual longbow) really exist, and virtual events (e.g. my shooting a grey cartoon raider with an arrow) really take place. Meanwhile, virtual irrealists hold that virtual objects do not really exist, and that virtual events do not really occur.

The virtual ir/realist debate concerns an intrinsically interesting ontological question, which also impacts surrounding debates about virtual reality. For example, since at least Nozick (1974), there has been debate about whether experiences undergone within a virtual environment are somehow not as valuable as real-life experience. Virtual irrealists have an ontological justification for this intuition; similarly, realists can naturally say that virtual experiences are (or at least can be) just as valuable are real ones. ${ }^{2}$ Relatedly, there are questions about whether perceptual experiences within virtual reality are non-illusory. Here, realism pairs well with the idea that our perceptions of them are veridical, and irrealism with the claim that such perceptual experiences are (something like) illusions. Finally, one area of rising interest concerns the (virtual) theft of virtual objects. ${ }^{3}$ On its face, these require adopting virtual realism; after all, you can't steal something that doesn't exist. However, virtual irrealists may be able to do some fancy footwork to explicate what is going on in such cases. For these reasons, those interested in virtual reality should pay some attention to this broadly ontological debate.

Recently, Chalmers (2017) has developed a particular version of

${ }^{1}$ Following Chalmers, we set this debate as one concerning the "reality" of virtual objects/events. But what does 'real' here mean? We, like Dummett (1963: 146), Putnam (1981: 49), Wright (1986: 5), Bennett (2009: 41), and Jenkins (2010), take being an F-realist to amount to (or, at minimum, involve) claiming that the way that the Fs are is mind-independent-i.e., independent of the way we think, talk, experience, or conceptualize, about the Fs. C.f. Chalmers 2009: 92 for an alternative conception, and Joyce 2009 for criticism of Chalmers.

${ }^{2}$ Obviously, this massively simplifies the issue here since one can, for example, be an irrealist and hold that virtual experiences are as valuable as real ones, or be a realist and claim virtual experiences are less valuable than non-virtual ones. For further discussion, see Cogburn and Silcox 2014 and Wildman (ms).

${ }^{3}$ For some recent discussion see e.g. Lodder 2013, Wall 2009, and Noveck and Balkin 2006. 
realism, virtual digitalism. Here, we aim to do two things. First, we will critique Chalmers' virtual digitalism — and, as our objections readily generalize to cover alternative versions, realism in general. Second, building off our critique, we go on to develop a broadly Waltonian version of virtual irrealism, virtual walt-fictionalism. This, we suggest, is a natural account of virtual objects.

More concretely, we begin (§1) by clarifying two forms of virtual digitalism - a strong version that identifies virtual and digital objects and a weaker version that rejects identification, though still plumps for a kind of dependence relation. We go on $(\S \S 2-3)$ to challenge these, before briefly dismissing $(\$ 4)$ a potential wrinkle. We then sketch (§5) an irrealist alternative, finally concluding (\$6) by briefly considering future avenues for developing this alternative picture.

\section{Two virtual digitalisms}

In its simplest form, virtual digitalism is the conjunction of:

(1) Virtual objects are digital objects

(2) Events in virtual worlds (largely) are digital events ${ }^{4}$

As a simplifying assumption we, following Chalmers, take digital objects to be data structures (2017: 317), which are themselves grounded in computational processes (that are in turn grounded in physical processes); we return to this assumption in (\$4).

There are several, conflicting ways to be a virtual digitalist, depending upon how one understands the 'are' in (1) and (2). If one treats the 'are' as expressing identity, the resulting position, strong virtual digitalism, then centres around a pair of identity theses:

(S1) Virtual objects are identical to (really existing) digital objects

(S2)Events in virtual worlds are identical to (really occurring) digital events

\footnotetext{
${ }^{4}$ We, following Chalmers, include the "largely" qualification in order to allow for events that are physical (e.g., users moving controllers around). We take a similar restriction applies in the case of (S2) and (W2) but, for readability, have left it suppressed.
} 
In contrast, one can read the 'are' not as identity, but rather as something like dependence. The resulting weak virtual digitalism holds that virtual objects/events are not identical to digital objects/events, though the former are in some sense dependent upon the latter. Thus, it is committed to:

(W1) Virtual objects are dependent upon distinct, really existing digital objects

(W2) Events in virtual worlds are dependent upon distinct, really occurring digital events

Different variants of weak virtual digitalism replace 'dependence' with alternative relations (e.g. grounding, supervenience, etc.), though the core idea remains the same: virtual objects are distinct entities whose (real!) existence is derived from the existence of more fundamental digital objects.

One can adopt various combinations of these principles; for example, it is coherent to accept an identity theory for virtual objects, but dependence theory for virtual events (i.e., to hold S1 and W2). Exactly how coherent these combinations are is an interesting question. However, the objections we will raise in the following can be readily applied to any combination of the above principles. So, for ease of presentation, we will focus on the "natural" packages described above.

It is not clear whether Chalmers himself prefers strong or weak digitalism, as he says things that appear to support both views. For example, he initially suggests that we can regard virtual objects 'as data structures'. This characterisation, given as a tentative 'first approximation' (2017: 317), is suggestive of a strong form of virtual digitalism. Further, the arguments he gives to motivate virtual digitalism - discussed below in $\$ 2.1$ - only make sense assuming strong digitalism. However, Chalmers also says that a virtual object is 'a higher-level entity constituted by ... data structures' (2017: 317), which suggests weak digitalism. Additionally, in earlier work, Chalmers explicitly rejected identifying virtual and digital objects:

... we should reject claims of token identity between microscopic and macroscopic levels. Tables are not identical to any object characterized purely in terms of quantum-mechanics; likewise, virtual tables are not identical to any objects characterized purely in terms of bits. But never- 
theless, facts about tables supervene on quantum-mechanical facts, and facts about virtual tables supervene on computational facts. So it seems reasonable to say that tables are constituted by quantum processes, and that virtual tables are constituted by computational processes. (MaM)

Regardless, it is clear that both strong and weak variants are available to would-be digitalists. Consequently, a thorough discussion of virtual digitalism must take both into account. And, more generally, any form of virtual realism that does not take virtual objects/ events to be sui generis entities but instead treats them as intimately bound up with some other class of entity (either via identity or via dependence) will also divide in this way. Thus, properly assessing the viability of virtual realism more broadly requires giving both strong and weak virtual digitalism serious consideration.

The proceeding two sections focus on them in turn. In "The Virtual and the Real" Chalmers first raised arguments against a fictionalist view of the virtual, then proceeded to defend his digitalist position. Here, we reverse this order: we first consider arguments against virtual digitalism (in both strong and weak forms), then propose a fictionalist position that can stand up to Chalmers' critique.

\section{Against strong virtual digitalism}

As detailed in the previous section, strong virtual digitalism (SVD for short) takes virtual objects to be identical to digital objects, and virtual events to be (largely) identical to digital events. Such a position is both prima facie appealing and fits well with many of things that Chalmers says in favour of virtual digitalism.

In this section, we sketch two problems for SVD. The first is broadly motivational: the arguments that Chalmers has offered for SVD fail. Consequently, there is - if you'll pardon the pun-no strong reason to accept SVD. Meanwhile, the second concerns the possibility of cross-platform play.

\subsection{Motivation problems}

Chalmers (2017) offers two arguments for virtual digitalism. The argument from causal powers states: 
(C1) Virtual objects have certain causal powers (to affect other virtual objects, to affect users, and so on.)

(C2) Digital objects really have those causal powers (and nothing else does)

(C3) Therefore, virtual objects are digital objects. ${ }^{5}$

This argument only makes sense if we read the 'are' in (C3) as an expression of identity; if it were something weaker, then (C1) and (C2) would be contradictory (how could only digital objects have the relevant causal powers if non-identical virtual objects also have such powers?).

Regardless, the argument is problematic. Proper virtual reality is interactive, in the sense that user actions can make significant differences to the virtual environment. ${ }^{6}$ Such interactivity requires that users have causal powers to affect virtual objects - otherwise, they wouldn't be able to interact. Yet users are not digital objects. ${ }^{7}$ Consequently, (C2) ought to be rejected.

In reply, the SVDist could claim that the argument wasn't intended to capture general causal powers; rather, it was meant to involve certain specific causal powers (e.g. the power to affect these specific virtual objects in such-and-such a manner). By definition, these powers are not available to users, so the objection to (2) is blocked.

However, this is not a satisfying response. First, restricting the argument to specific powers in this way also restricts the overall upshot - at best, the argument can now tell us that a particular virtual object $v$ is identical to a particular digital object $d$ in virtue of the fact that only $d$ possesses the specific power $F$ that $v$ is thought to have. This radically restricts the scope of SVD; it now only extends to those virtual objects for which we can specify such hyper-specific

${ }^{5}$ Chalmers 2017: 318.

${ }^{6}$ Chalmers 2017: 312.

${ }^{7}$ Objection: What if we're all in the Matrix? Then users are digital objects! Reply: In that case, users are a radically different kind of digital object than the sort relevant here. For lack of better terms, users would be Matrix-external digital objects, in contrast to our Matrix-internal digital avatars which engage with Matrixinternal digital objects. 
powers. Second, it is unclear what specific powers we might invoke that would allow us to successfully run the argument. This is primarily because doing so requires that we naturally ascribe the same power to both the virtual and the digital object. ${ }^{8}$ However, in purely virtual terms, we might say that virtual object $v$ has the power to make virtual ball $b$ virtually blue, while we might also say that digital object $d$ has the power to make digital object $d 2$ have property $G$. The trouble is that there is no obvious reason to say that these are the same powers, rather than different ones - in fact, assuming so without external motivation begs the question, since it assumes that a specific digital outcome is identical to a specific virtual outcome.

The second argument for strong virtual digitalism is the argument from perception:

(P1) When using virtual reality, we perceive (only) virtual objects.

(P2) The objects we perceive are the causal basis of our perceptual experiences.

(P3) When using virtual reality, the causal basis of our perceptual experiences are digital objects.

(P5) Therefore, virtual objects are digital objects. ${ }^{9}$

Note that, as with the previous, this argument is only plausible if the 'are' in (P4) expresses identity; if it were a weaker relation, then (P2) and (P3) would entail the falsity of (P1).

However, this argument also fails. First, the argument is only valid if we understand (P3) as an exhaustive claim:

$\left(\mathrm{P}^{*}\right)$ When using virtual reality, the causal basis of all our perceptual experiences are only digital objects.

Without 'all' and 'only' we leave open the possibility that some of our VR perceptual experiences do not have just digital objects as their causal basis; consequently, the virtual objects involved in these

\footnotetext{
${ }^{8}$ This second objection would seem to apply even if there is no restriction to specific causal powers. We thank an anonymous referee for pointing this out.

${ }^{9}$ Chalmers 2017: 318.
} 
experiences are either identical to some non-digital objects or are sui generis entities. So, (P4) only follows if we read the argument with ( $\left.3^{*}\right)$ instead of (3).

Problem: (P3*) is false. Consider a multi-user VR environment. Within this environment, the causal basis for at least some of our perceptual experiences will be other users. Consequently, it is not the case that our VR perceptual experiences have only digital objects as their causal basis.

Thus there is a kind of dilemma: either the argument features (P3) and is invalid, or features ( $\left.\mathrm{P}^{*}\right)$ and includes a false premise. Either way, the argument fails.

SVDists might reply by arguing that multi-user VR environments are radically different than single-user environments. Two replies. First, this looks utterly ad hoc. Put bluntly, the fundamental ontological nature of virtual objects shouldn't depend upon how many users are engaged with them. But second, single-user environments work just as well. Consider the case where you look at your virtual self (e.g. your avatar) moving around. Given (P2), in so doing, you see yourself. However, you are not a digital object. Consequently, $\left(\mathrm{P} 3^{*}\right)$ is false. ${ }^{10}$

When we combined the failure of this argument with that of the previous, we can see that SVD has a motivation problem: what has been said in favour of it looks problematic, leaving it unclear why we should adopt the position. This suffices to call into question the viability of SVD.

Of course, it's one thing to attack the positive arguments in favour of a position and quite another to raise objections to it. With that in mind, the next sub-section offers a problem that seems to undermine strong virtual digitalism entirely.

${ }^{10}$ Chalmers might attempt to respond to this point by restricting (P2) to proximal, rather than distal causes. (Thanks to an anonymous referee for suggesting this reply.) However, so restricted, (P2) looks implausible. For consider a case where Nathan and Neil are using Skype to video chat. It is plausible to say that when Nathan looks at the image on his screen, he perceives Neil (though Nathan is in Tilburg and Neil in Glasgow). Yet this is incompatible with the restricted version of (P2). Further, this restriction is incompatible with Chalmers' interpretation of mirror cases (2017: 328-9), since it entails that we don't perceive other cars when we look in our rear-view mirrors. 


\subsection{The cross-play problem}

Cross-play occurs whenever users on multiple, distinct systems interact within a single virtual environment. Though few apps supporting cross-play are available for VR so far, they do exist (as in the case of AltspaceVR and Spaces from Facebook), and can be expected to expand as VR becomes a social platform. Further, cross-play has occurred in non-VR cases: for example, Epic Games' Fortnite (briefly) allowed players on Xbox and PlayStation consoles to play together (once it was discovered, it was quickly quashed by Sony executives), and Rocket League is poised to support wide-spread cross-play between Xbox, PlayStation, Nintendo Switch, and PC users. ${ }^{11}$

The possibility of cross-play cases raises a significant problem for SVD. Suppose that Frisbee is a cross-play-supporting VR application of a virtual environment wherein multiple users can play virtually throw around a virtual frisbee. Suppose further that Neil, on his HTC Vive in 32-bit mode, and Nathan, using his Oculus Rift (which only supports 64-bit mode), are playing Frisbee together over a peerto-peer network (i.e. there is no mediating server that controls the game). In such a case, there is a single virtual object that Neil and $\mathrm{Na}$ than are both engaged with - the virtual frisbee. However, as the local hardware and system architecture of their respective VR set-ups are radically different and, since there is no common server, there is no single digital object that both Neil and Nathan are engaged with. Consequently, there is no digital object that the virtual frisbee is identical to. In other words, strong virtual digitalism is false. ${ }^{12}$

More generally, the possibility of VR cross-play entails the possibility of users co-engaging with a single virtual object despite there being no single digital object they are co-engaged with. Consequently, it is possible that these virtual objects are not identical to the relevant digital objects. Given the necessity of distinctness, it follows that the former are not identical to the latter, which entails the falsity of SVD.

\footnotetext{
11 http://www.ign.com/articles/2016/07/20/rocket-league-ps4-xbox-onecross-network-play-now-ready-for-release-only-needs-sonys-approval

${ }^{12}$ In effect, this cross-play problem for strong virtual digitalism parallels multiple-realizability objections to mind-brain identity, so the objections and replies in that debate will, mutatis mutandis, arise here.
} 
We anticipate two potential responses. The first is to claim that cross-play is far from typical, and so not an appropriate test case for theorizing. However, whilst VR cross-play is not typical today, the explicit ambition of Facebook (the owners of Oculus) is to make it a new social arena, with widespread uptake across multiple platforms. ${ }^{13}$ So cross-play will not be atypical for long.

The second response is that what is happening in a cross-play case is actually two distinct, albeit corresponding experiences, involving two digital and two virtual objects. By analogy, consider chess by mail; here, each player acts out the moves on their own board, separately communicating their moves to their opponent. Here you have two black kings, two white queens, and, one might say, two games of chess-games that match each other in the competitive details, but two nonetheless.

In a similar vein, according to this response, cross-play involves two distinct, but corresponding, virtual worlds, populated by distinct, corresponding virtual objects - Neil's frisbee and Nathan's frisbee - which are identical to distinct digital objects: the digital object on Neil's rig and the digital object on Nathan's set-up. Duplicating virtual objects in the manner would dodge the objection: there is not a single virtual object that Neil and Nathan are both interacting with, so there is no problem.

But such rampant duplication is a repugnant result. Sticking with the chess analogy, it is plausible that while two sets of objects/boards are being used, they clearly represent a single game, with a single outcome. The "game-object" that is (say) the Black queen is not identical to the carved piece of wood on Neil's board, nor the Lego figure on Nathan's; rather, it is something abstracted from the specific physical tokens used to represent it. Similarly, the causal interactions that make up the game are, properly understood, not duplicated: Nathan moves one queen, not two (otherwise, he has cheated), and we record one win, not two (else best-out-of-three would cease to make sense).

These points equally apply in the VR case: two systems are being used, but they represent a single virtual world, featuring a single virtual frisbee. The representational modes of playing chess without

${ }^{13}$ See e.g. https://www.engadget.com/2017/12/19/facebook-spaces-vive/ and https://www.engadget.com/2017/04/19/facebook-spaces-social-vr/ 
a board make it clear that the game is not to be identified with any given board, and the cross-play VR cases make it clear that the VR game (or the objects therein) are not to be identified with the processing bases. To do so is akin to mistakenly equate a prop-e.g. the carved piece of wood/Lego mini-fig — with the "game-object" - the Black queen - that they represent.

\section{Against weak virtual digitalism}

The alternative version of digitalism takes virtual objects to be distinct from, through dependent upon, digital objects. This weak virtual digitalism (WVD, for short), retains the key aspect of virtual digitalism — virtual objects/events really exist — but avoids collapsing the distinction between the virtual and the digital.

In this section, we critique WVD. Specifically, we target the fact that WVD requires that virtual entities stand in genuine causal relations. For Chalmers, this is a consequence of his 'philosophical structuralism', whereby virtual and non-virtual reality are ultimately different implementations of closely related causal structures (2017: 349). Even non-structuralist versions of WVD, should they emerge, will incur a similar commitment, on pain of rendering the virtual merely epiphenomenal, and so violating Alexander's Dictum, i.e. to be is to have causal powers.

In $\S 3.1$, we give reasons for thinking that virtual entities do not stand in such relations, and hence for the falsity of WVD. However, even if WVDists were able to demonstrate that the virtual involves genuine causal relations, this leads to a second objection, discussed in $\S 3.2$ : a form of the exclusion problem. The upshot is that no matter whether virtual entities do or do not stand in genuine causal relations, WVD should be rejected.

\subsection{Causes and pseudo-causes}

Salmon (1998: 194), citing Reichenbach (1958), points out that there are some processes which seem causal, but in fact are not. His classic example is that of the spotlight moving along the wall. Each point where the light lands on the wall is followed by an adjacent point where the light lands next, giving the impression of a genuine causal 
process relating the two. However, with a distant-enough wall, the spotlight could 'travel' across the wall at a speed faster than light. As this is physically impossible, it becomes apparent that the spotlight's 'journey' is a pseudo process: it has the appearance of an ordinary causal process ${ }^{14}$ but not the requisite character of one.

A more salient example of pseudo causal processes is traditional animation. Jerry strikes Tom on the head, and a lump emerges on Tom's head. We naturally describe this as a case where the strike caused the lump. Of course, there is no genuine causal relation between the frames of the animation. There is a relevant causal relation nearby, however, involving the animator. She draws this sequence because it fits the story she is trying to tell, and that story roughly mirrors how things happen in the world: hits on heads result in lumps on heads. The genuine causal relation lies not between the frames of the animation, but rather between the thoughts that lie in the head of the animator.

With these examples in mind, we can ask: are the causal interactions between virtual entities genuine causal processes as WVD requires, or pseudo processes like those of the spotlight and of traditional animation?

The first point to make is that VR is unlike traditional animation in at least one crucial respect: in traditional animation, each frame's relation to the next is set by the intentions of the animator, which may or may not respect ordinary causal interactions in the world. In modern-day VR, on the other hand, typically a game engine controls the production of frames, doing so on the basis of an underlying physics engine that encodes (roughly) real-world physics. Thus, whilst the animator may decide on a whim that the ball will bounce this way or that, regardless of the physics, the game engine sticks to a set of pre-ordained rules about how things interact.

This should not make us think that there is a genuine causal relation between the occurrences in VR, however. The game engine is doing nothing more than the animator in this case. The VR experience may be being produced from a digital process rather than an organic one, but the genuine causal interactions are between the

\footnotetext{
${ }^{14}$ The internet is replete with examples of cats chasing laser points in a way that suggests they are readily taken in.
} 
execution of bits of code that decide which frames to render, not between the rendered frames themselves. VR and traditional animation are on a par in this respect.

More importantly, one characteristic feature of VR is interactivity - users can fire a gun, kill a zombie, and thereby feel relieved through VR. This kind of causal interaction is not possible with traditional animation which is drawn in advance, and represented in a pre-determined way. The causal chain between the user, a virtual gun, a virtual zombie, and the user again would seem to entail that the virtual causal steps were indeed genuine.

But while it is certainly clear that a genuine causal interaction can be traced from the user, via the controller, to the computer, and back to the user via the headset, nowhere in that chain does a gun or a zombie appear. This is despite the fact that talk of guns and zombies is clearly an entirely sensible way to describe, report, and discuss the VR experience that took place. Yet this is just loose talk. For compare the obvious way of describing what happened in traditional animation: Jerry smacked Tom on the head and caused a lump to appear. Or how we talk of the spotlight 'traveling' across the wall. These are intuitively appealing ways of apparently characterising a causal process that nevertheless reifies a pseudo process instead. And we need independent reason to think that the causal interactions in VR are genuine, rather than simply pseudo. None seem forthcoming.

\subsection{Is virtual causation genuine enough?}

We accept that one genuine causal process can be traced from the user, via controller, $\mathrm{PC}$, and headset, back to the user again. Of course, that does not mean that there is not also a second, corresponding, equally genuine, causal process simultaneously holding between the user, a gun, a zombie, and back to the user again. Proposing a second such causal chain - one that supervenes upon, but does not wholly reduce to, the digital base - allows the WVDist to retain both dependence (without reduction!) of the virtual upon the digital, and genuine causal relations between virtual objects and events.

In the philosophy of mind, non-reductive physicalism (NRP) is the view that the mental supervenes on, but does not reduce to, the physical. It has been argued - by Jaegwon Kim (1993) in 
particular - that NRP is incompatible with three other attractive theses: first, that the physical domain is causally closed, second, that the mental has causal efficacy, and third, that there is no systematic causal redundancy. Kim's 'causal exclusion argument' says (roughly) that if we assume the mental has causal efficacy, and if we assume (as physicalists must) that the physical domain is causally closed, then either the mental is identical with something physical, or there is systematic causal redundancy. Adding the further assumption that there is no such redundancy, we are left with the conclusion that the mental is identical with something physical, pace NRP.

There is an obvious parallel between WVD and NRP: they both claim that one domain depends upon, but does not reduce to, another, but that the dependent domain has causal efficacy. This suggests that there will be a causal exclusion problem for WVD, akin to that faced by NRP: the digital (non-virtual) process does all the causal work, leaving none for the virtual processes to do. More precisely, we give a causal exclusion argument against WVD (modelled on Zhong's 2014 version of the traditional exclusion argument):

(E1) Virtual properties supervene on non-virtual properties. [Central tenet of WVD]

(E2) Virtual properties are not identical with non-virtual properties. [Central tenet of WVD]

(E3) If an instance of a non-virtual property has a cause that occurs at $t$, it has a (sufficient) non-virtual cause that occurs at $t$.

(E4) It is not the case that virtual and non-virtual properties causally overdetermine the effects (in a systematic way).

(E5) Therefore, virtual properties are causally inefficacious. [Contradiction of WVD]

In this argument (E1) and (E2) are part of the definition of WVD, and the conclusion flatly contradicts the causal commitments of WVD. The argument appears to be valid ${ }^{15}$ and so the cogency of

${ }^{15}$ The validity will depend, at least in part, on deep issues to do with the metaphysics of causation, and the notion of a sufficient cause. We side-step those here. 
WVD depends on the plausibility of (E3) and (E4).

Premise (E3) is a port of the causal closure principle from the NRP debate. In the NRP version, the idea is that every physical event has a sufficient physical cause. ${ }^{16}$ More loosely, the (fundamental) physical domain does all the necessary causal work. Once ported into the argument for WVD, the closure principle becomes about the virtual and non-virtual instead. In this form the closure premise claims that there is suitably complete causal history of our actions when using VR that relates only non-virtual things (the CPU, GPU, headset screen, retina, optic nerve, etc.). One need not deny that there is a good way to describe what happened that doesn't mention the processors, screen etc. (e.g. 'shot a gun and killed a zombie') to think that this principle is plausible. This seems exactly analogous to the sense in which we may talk of ordinary macro-causal connections between, say, billiard balls, and yet still accept that the entire process could be re-described in terms of fundamental micro-physical interactions. If we accept that the microphysical story suffices in such cases, then it seems plausible to accept that the non-virtual story (macro or micro) suffices in the VR cases too.

A classic line of argument in favour of (E4) runs as follows: when a firing squad executes a prisoner, the death of the prisoner is overdetermined in the sense that there were several distinct, and equally sufficient, causes of that death present (assuming each member of the squad was deadly accurate). This is a literal co-incidence, and an entirely plausible scenario to boot. However, systematic overdetermination of the sort that would need to obtain for the NRPist, or the WVDist, is not of this form: it does not posit some isolated co-incidences here and there, but ubiquitous and systematic co-incidence in every instance of mental/virtual causation. Such systematic overdetermination is widely claimed to be implausible (Kim 1993, Merricks 2001, Papineau 2002, Schiffer 1987, Zhong 2014).

If we accept both (E3) and (E4), then we get the result that the non-virtual alone does sufficient causal work, and that there is not any systematic causal over-sufficiency in the world. Thus, there is no causal work for the virtual left to do.

${ }^{16}$ This is a comparatively weak formulation of the causal closure principle. 


\subsection{Responses from the weak virtual digitalist}

The parallels between WVD and NRP prompted our formation of the above exclusion argument, but that parallel also opens up a range of extant responses that the WVDist might adopt in response.

Non-physicalists can, and do, deny the causal closure principle in the original NRP exclusion argument. One might think: perhaps the WVDist can similarly deny (3). Whilst denying closure is obviously a reasonable position for the non-physicalst to take, it is far from obvious that a physicalist - who the Kimian exclusion argument is directed at - can do the same and still be a physicalist in the ordinary sense. The digitalist faces the same problem: what makes them a digitalist is the commitment to the idea that underpinning each virtual object or event is some digital process that realises it. Accepting that some virtual event did causal work, but that no non-virtual, digital, event did — as denying the closure premise requires — drives a wedge between the virtual and the digital that no genuine virtual digitalist could accept.

Another standard approach to the original causal exclusion argument is to query the application of overdetmination premise. For example, any given biological process (digestion, say) has a parallel chemical process that underpins it. And any given chemical process is further underpinned by fundamental physical process. There are therefore three causal processes we can tell describe when one suffers acid reflux: one biological, one chemical, one physical. If these three descriptions are rivals, then the overdetermination principle presses us to choose between them on pain of positing an overabundance of causal structure in the world. As such the exclusion argument proves to much: it eliminates biological causes and chemical causes from the world! This reductio demonstrates that something has gone wrong in the exclusion argument. Notice, however, that if the three processes are not rivals after all, then the overdetermination principle is silent on them. Conceived as re-descriptions of the same phenomena (fundamentally physical or not), the biological, chemical, and physical casual stories are no longer in tension. The overdetermination principle might be true, but it simply does not apply in this case. ${ }^{17}$

${ }^{17}$ We remain judiciously silent on whether this case is sufficiently analogous 
Such a response would appear to save chemistry and biology from exclusion, so can the same approach be used by the WVDist? First, the saviour of chemistry and biology in this example appealed to the fact that they were simply describing the same phenomena, in a different way. That is highly suggestive of a reductive position that the WVDist simply cannot adopt in the virtual-digital case, lest they collapse their position into strong virtual digitalism. Second, defenders of chemistry and biology do not claim that with each new level of causal process comes a genuinely novel reality. The WVDist, on the other hand, requires that the advent of the right digital processes and human interaction, gave rise to a genuinely novel reality. This is a key dis-analogy between the two cases.

\section{Ironing out a potential wrinkle}

The foregoing lays out the argument against two distinct versions of virtual digitalism. However, there is a third way. ${ }^{18}$ As we understand them, both strong and weak virtual digitalism concern the relationship between virtual and digital objects; the former identifies the two, the latter takes the virtual to depend upon the digital. But neither specifies the relationship between digital objects and data structures. Instead, we earlier adopted, as a simplifying assumption, the idea that digital objects just are data structures. The trouble is that it is possible to weaken this relation, taking digital objects to distinct from, but dependent upon, data structures. In other words, along with adopting either a strong or weak account of the relationship between virtual and digital objects, one can also adopt a strong or weak account of the relationship between digital objects and data structures. And what option we take concerning the latter relationship seems to have some interesting knock-on consequences. Specifically, given a weak position regarding the relationship between digital objects and data structures, the cross-play problem appears to evaporate: assuming that the relation between virtual objects and digital objects is that of identity, but that the relation between the digital objects and their associated data structures is something weaker (e.g.,

to the original mental-physical case to be persuasive.

${ }^{18}$ Thanks to David Chalmers for pushing us on this line. 
dependence), then we can say that there is just one (virtual) frisbee, identical to one (digital) object, and this digital object is itself dependent upon distinct data structures.

That said, this doesn't help the digital realist. For adding this extra layer gives rise to a dialectic parallel to that outlined above. Instead of asking, "are virtual objects real?", we now ask, "are digital objects real?". Everyone should grant the reality of the data structures on the processors, ${ }^{19}$ but the reality of digital objects is very much up still up for debate.

If the data structures are identical with the digital objects - a view we can dub strong digital realism - then the answer to that question would be 'yes', and, depending upon which option one takes regarding strong or weak virtual digitalism, our above objections kick in.

However, if data structures and digital objects are distinct, then the question is not yet settled: an argument needs to be given for weak digital realism. And, more importantly, the causal arguments against WVD apply, mutatis mutandis: the weak digital realist has no obvious way to show they are employing a genuine, rather than pseudo-, notion of causation at the level of digital objects, and the view faces a version of the exclusion problem.

So, to come back to the position detailed three paragraphs ago, identifying virtual objects with digital objects while having digital objects merely depend upon data structures avoids the cross-play problem frying pan, but does so by jumping directly into the (one level down) fire of exclusion problems and pseudo-causation.

Setting this aside, however, note that, absent an argument for weak digital realism, the reality of virtual objects is not settled by identifying them with digital objects. For while this third way may offer an escape from the cross-play problem, it does so at the cost of rendering Chalmers' case for the reality of virtual objects incomplete at best.

${ }^{19}$ The idea of rejecting the existence of data structures due to philosophical reasons is laughable. To paraphrase Lewis, (1991: 58-9), how would you like the job of telling the programmers that they must change their ways, and abjure countless errors, now that philosophy has discovered that there are no data structures? 


\section{Virtual walt-fictionalism}

We have argued that virtual digitalism can come in (at least) two forms, strong and weak. The strong version is, we have argued, too strong: there are compelling cases wherein a single virtual entity is supposedly identical to two distinct digital entities. Meanwhile, the weak option is too weak: virtual causation is either a pseudo-process, unfit to sustain the causal commitments of the picture proposed, or is excluded in favour of its digital base. The upshot is that virtual digitalism as a whole looks unpromising. And, as virtual digitalism is the best form of virtual realism available, the same goes for it as well. We need to consider the alternatives.

In this section, we do exactly that, sketching virtual walt-fictionalism, a form of irrealism. We begin ( $\$ 4.1)$ by spelling out the background theory, Walton's conception of fictionality. Having fixed this foundation, we then (\$4.2) proceed to explicate our account of virtual reality. Finally (\$4.3), we consider the benefits of adopting this picture.

\subsection{Background: Walton on fiction and fictionality}

Walton offers an account of representational (art)works founded upon a theory of make-believe.

According to Walton, when we engage with representational artworks, we play sophisticated games of make-believe. These games are not different in kind from those games played during childhood (cops and robbers, bears, etc.); all that is different is (perhaps) level of sophistication and particular imaginative activities.

More specifically, according to Walton, when we engage with a fiction like Harry Potter, we imagine (i.e., make-believe) that there are witches and wizards, that Hermione is one, that she goes to a school named 'Hogwarts', etc. But the contents of these imaginings are not chaotic; rather, they are 'constrained ... some are proper, appropriate in certain contexts, and others not.' (Walton 1990: 9). In particular, our imaginings are constrained by the objective features of the work in question (and perhaps wider features of the context of creation and critical appreciation). This is not to say that one cannot engage with Harry Potter and imagine e.g. that Voldemort was in fact a selfless hero, Harry a terrible villain, and the story suggests 
otherwise because history is written by the victors. Rather, it is to say that not all Harry Potter-based games of make-believe are created equal. Neil, who images that Harry is a heroic figure and Voldemort a wicked villain, is playing a game that is authorized for Harry Potter, whereas Nathan, who imagines that Voldemort was the real victim, is playing a game that is unauthorized. More specifically, in conducting his game, Nathan is ignoring or mis-interpreting certain objective features of the work, leading to his deviant, unauthorized (but still perfectly possible to play!) game of make-believe.

To give the proper Waltonian gloss, representational artworks are props - items whose existence and real features are used to guide and determine features of the imagined world of the game of makebelieve. Many things are designed props - for example, dolls and toy trucks are designed specifically to be employed as props in specific games of make-believe, as are (according to Walton) fictional novels and paintings. And nearly everything can be an ad hoc prop, 'pressed into service for a single game of make-believe on a single occasion' (Walton 1990: 51). So, for example, a stump can serve as a prop in a particular game of make-believe according to which we are to makebelieve that, wherever there is a stump, there is a bear, while a paper towel roll makes a serviceable spyglass, despite the fact that this is not what it was intended for.

Props are items whose existence and features are used to guide and determine the contents of a particular game of make-believe. ${ }^{20}$ And what tells us how to 'translate' the relevant features of props into the appropriate imaginative contents are principles of generationroughly, "rules" that prescribe what it is we are to imagine (makebelieve). These principles can take various forms. For example, they can be conditional, saying that if a certain actual feature is noted, then we should imagine something - for example, 'if there is a mug on the table, then imagine there is a goblin on the table'. Alternatively, some are straightforwardly stipulative; e.g., 'Imagine that there is a goblin on the table'. Finally, some are supplemental, bolstering or clarifying

${ }^{20}$ Note that props can 'stand-in' for themselves - a particular person might serve as a "prop" for themselves (e.g., Nathan might be a prop for Nathan himself). Additionally, props are not restricted to objects - for example, Nathan's running away from a tree stump can serve as a prop, indicating that, within the relevant game of make-believe, Nathan is running away from a bear. 
other principles-e.g., 'if we are prescribed to imagine a goblin on the table, then imagine that it is wearing a red hat'.

In this way, according to Walton, our engagement with fictions is both imaginative, in the sense that being so engaged involves imagining (make-believing) that certain things are the case, and structured, in the sense that there are oftentimes rules that determine what is/ is not to be imagined (e.g. we might be prescribed to imagine that $x$ is dead if, in reality, $x$ is lying motionless on the ground, or that $x$ is a bear if, in reality, $x$ is a tree stump).

Taking all of this together, Walton says that a fiction is a representational work that has as one of its functions the role of serving as a prop in a specific game of make-believe (1990: 51). One upshot of this definition is that 'fiction' will apply to many things we naturally tend to not call fictions_- dolls, dances, and disco songs, as well as novels, paintings, and plays. As Walton is explicit that he is not interested in capturing the everyday usage of 'fiction' (1990: 70), this is a feature, rather than a bug. That said, to avoid potential confusion, following Friend (2008: 154), we use the term 'walt-fiction' to pick out this category of works.

Relatedly, a proposition $\mathrm{p}$ is fictional in work $\mathrm{w}$ iff $\mathrm{w}$ has the function of being a prop in a game of make-believe with principles of generation that entail, given the features of $\mathrm{w}$, that full appreciation of w requires imagining that $\mathrm{p}$. Or, slightly simpler, 'a proposition is fictional just in case there is a prescription to the fact that it is to be imagined' (Walton 2013: 9). ${ }^{21}$

\subsection{Stating the view}

The central claim of virtual walt-fictionalism (VWF) is that virtual reality is a kind of walt-fiction, and our engagement with VR is not different in kind from our engagement with other forms of walt-fiction.

In particular, proper engagement with VR is a kind of make-believe, featuring VR specific props and principles of generation. These VR specific props include digital elements like the particular images, sounds, and haptic feedback mechanisms employed by VR systems.

\footnotetext{
${ }^{21}$ For further discussion of Waltons' account of fictionality, see e.g. Woodward 2014 and Wildman and Woodward 2018.
} 
Such elements really exist - we see, hear, and feel them whenever we engage with VR applications, there are a multitude of individuals within the VR industry who create and produce them, and legions of individuals who criticize them. Further, the features of these elements serve to characterize the various games of make-believe that we play when we engage with a particular VR application, via the appropriate VR-specific principles of generation.

For example, take Sansar, Linden Lab's VR-version of Second Life. Some Sansar-specific principles of generation are

(i) Imagine that this projected image of a large, green, ogrelooking thing is a fully 3 -dimensional entity;

(ii) If the text of an English sentence proceeded by '[Gørbl@rg]:' appears in the window in the bottom right, then imagine that this ogre-thing uttered the relevant sentence out-loud.

(iii) If we are proscribed to imagine that this thing uttered something, then imagine that Nathan (whose avatar the ogre-thing is) said it.

Assuming VWF, it is clear that the props-i.e., the code, images, sounds, etc. that are used in order to engage in the relevant game of make-believe - are real (otherwise, it would be impossible to be able to play the associated game). However, these props are not identical to the virtual objects. That's because, for the VWFist, virtual objects are those objects that we make-believe to exist whenever we engage with a particular VR walt-fiction in an authorized manner. ${ }^{22}$ Similarly, the reality of said props does not entail the reality of distinct virtual objects that depend upon the former for their existence. Instead, these virtual objects "exist", in as much as they do, only when we play the relevant games of make-believe. Consequently, they lack the robust reality that props possess.

For example, returning to Longbow, the VWFist will happily grant that images of little grey men wearing Viking helmets, castle

${ }^{22}$ Potential exception: there are some objects that appear within a virtual environment which represent themselves. In these cases, it is possible that the prop is identical to the "virtual object". However, these don't offer much hope for would-be realists. 
gates, balloons, arrows, and a bow are real, and that these images serve as props in a game of make-believe. But the little grey men wearing Viking helmets aren't real. Rather, they only "exist" within the Longbow game of make-believe. Once we stop playing the game, they stop "existing". ${ }^{23}$

Further, consider the various events involved in playing Longbow: pulling back the bowstring, firing an arrow, etc. These events, according to VWF, do not really occur. Instead, they fictionally occurthat is, we make-believe that they occur as part of the elaborate game of make-believe we engage in whenever we play Longbow. Of course, many of these events will roughly correspond to real-world actions that do really occur - for example, whenever Neil fictionally draws the bowstring (which doesn't really happen), he moves the control sticks in such-and-such a manner (which really does happen). For those who are conversant with the relevant game of make-believe, we can readily call the really occurring event 'their drawing the bow', but this is to use a fictional gloss to describe a distinct, nonbow-involving, really occurring event.

\subsection{The advantages of going Waltonian}

Virtual walt-fictionalism has a lot going for it. Perhaps the best point in its favour is that it is a natural extension of a powerful and intuitive general account of representational works.

Another favourable point is that VWF avoids the problems facing virtual digitalism. In particular, the causal powers argument stemmed from the idea that, within virtual environments, virtual objects appear to have specific causal powers; this was then used to motivate the idea that virtual objects are identical to digital objects, which are the seat of these powers. According to VWF, virtual objects fictionally have such causal powers - that is, within the relevant games of make-believe, these objects are taken to (exist and) possess the relevant powers. And this will be explained by some relevant principle of generation linking the content of our make-believing to some real feature of a relevant prop (or, depending on the kind

\footnotetext{
${ }^{23}$ Indeed, one might go further and simply deny that the little men exist at all. However, we are here happy to adopt the slightly weaker position.
} 
of principle, possibly simply stipulating it). Thus it is fictionally the case that Pikachu has the power to thundershock Squirtle because the various props that underpin our Pikachu-imaginings have certain powers that affect the props that underpin Squirtle-imaginings.

Similarly for the argument from perception. Chalmers' idea was that, when we are engaged with VR, we perceive virtual objects; this, in combination with the general perceptual rule that what we perceive is the causal basis of our perception, was meant to push us into identifying virtual objects with digital objects. According to VWF, this argument conflates fictional perceptions - i.e., what is perceived within a game of make-believe - with actual perceptions. If we consider what we are in fact perceiving - the images, sounds, and haptic feedback we are receiving - then the general perceptual principle will push us to say that we are perceiving the relevant props. Given our engagement with the relevant fiction, we can readily describe this in terms of what we, within the relevant game of makebelieve, are fictionally perceiving: that Jerry has hit Tom, resulting in a bump. In so doing, we focus not on what is in reality causing what we are seeing, hearing, etc., but rather on what is causing it in the world of the fiction. And, within the fiction, it is appropriate to say that it is the relevant virtual objects that we are perceiving. But this certainly does not entail that we are in fact perceiving the relevant virtual objects!

Relatedly, VWF is perfectly compatible with the possibility of cross-play. Principles of generation can map multiple, distinct props to the same "game-object" - this sort of thing is common-place in play-by-mail chess, role-playing-games, and serial fictions (for example, if we consider the whole Star Wars cannon, how many props are there for Obi-Wan Kenobi?). Adopting VWF makes VR cross-play simply another instance of a familiar occurrence.

VWF also avoids the various problems we raised against WVD. By denying that virtual events really occur (instead, they fictionally occur), the VWFist avoids postulating any sort of genuine causal relation between the virtual objects/events. Consequently, they need not worry about distinguishing genuine from pseudo-causation, nor about overdetermination.

Finally, it is worth discussing what VWF has to say about the phenomenology of virtuality. Naïve VR users, on this view, are engaged in 
an imaginative activity - they are playing a (rich, prop-driven) game of make-believe. Of course, because they are new to this game (and because the props are pretty exciting), they might mistakenly think that what they are perceiving is occurring in physical space. (In that sense, they are not unlike children who first encounter a puppet, and think that it is alive.) Meanwhile, experienced VR users are also engaged in the imaginative activity of playing a game of make-believe, and will have moved passed this sort of mis-conception. Further, because they are so well versed in these games, they will treat the 'virtual objects' they encounter in ways that wouldn't occur to the naïve users - they will learn to exploit 'distinctive affordances' (Chalmers 2017: 331) that the principles of generation license. And as such users become so intimately familiar with playing these games of make-believe (especially when these games are persistent and highly immersive), such users might (mistakenly!) start to think that the game worlds are a distinct kind of reality. Indeed, the way that we talk about fiction tends to encourage this mis-conception. But a mis-conception it is.

\section{Conclusion}

We began with an ontological question: are the objects and events that appear to make up the environments of virtual reality *real*? The virtual realist says they are, the irrealist that they are not. In an attempt to adjudicate this debate, we focused on the most developed form of virtual realism: Chalmers' virtual digitalism. After disentangling some details of the position, we argued that both weak and strong varieties of virtual digitalism face significant problemsproblems that push us towards the irrealist camp.

Building on this, we then proceeded to sketch virtual walt-fictonalism, a form of virtual irrealism based upon Walton's theory of fictionality. This position, we contend, provides a highly plausible account of the virtual, one which avoids the hazards that befell the digitalist.

Of course, these benefits might not be enough to sway the wouldbe virtual digitalist/realist to come over to the irrealist camp. No doubt there will be objections to virtual walt-fictionalism that must 
be addressed. ${ }^{24}$ but given the apparent advantages over the digitalist that we have laid out here, we think that virtual walt-fictionalism deserves to be taken seriously.

Neil McDonnell Philosophy

University of Glasgow neil.mcdonnell@glasgow.ac.uk

Nathan Wildman Tilburg Center for Logic, Ethics, and Philosophy of Science

Tilburg University N.W.Wildman@uvt.nl

\section{References}

Bennett, K. 2009. Composition, colocation and metaontology. In Metametaphysics: New Essays on the Foundations of Ontology, ed. by David John Chalmers, David Manley and Ryan Wasserman. Oxford University Press, pp. 38-76.

Chalmers, D. J. 2003. The matrix as metaphysics. Online at thematrix.com. Published in print in Philosophers Explore the Matrix, ed. by C. Grau. Oxford University Press, 2005.

Chalmers, D. J. 2009. Ontological anti-realism. In In Metametaphysics: New Essays on the Foundations of Ontology, ed. by David John Chalmers, David Manley and Ryan Wasserman. Oxford University Press, pp. 77-129.

Chalmers, D. J. 2017. The virtual and the real. Disputatio 9(46): 309-52. DOI: https://doi.org/10.1515/disp-2017-0009.

Cogburn, J.; and Silcox, M. 2014. Against brain-in-a-vatism: On the value of virtual reality. Philosophy and Technology 27: 561-79.

Dummett, M. 1978. Realism. In his Truth and Other Enigmas. London: Duckworth, 145-65.

Everett, A. 2013. The Nonexistent. Oxford University Press.

Friend, Stacie. 2008. Imagining fact and fiction. In New Waves in Aesthetics, ed. by Kathleen Stock and Katherine Thomsen-Jones. Palgrave-Macmillan. pp. $150-169$.

Heim, M. 2000. Virtual Realism. Oxford University Press.

Jenkins, C. S. 2010. What is ontological realism? Philosophy Compass 5 (10):880-90.

${ }^{24}$ For example, the VWF-er needs to provide an account of so-called virtual theft, as well as a general story about how to understand talk purportedly about virtual objects. For some steps towards this issues, see e.g. Everett 2013. 
Joyce, R. Moral anti-realism. In The Stanford Encyclopedia of Philosophy (Summer 2009 Edition), ed. by E. Zalta. 2009. URL http://plato.stanford.edu/ archives/sum2009/entries/moral-anti-realism/

Kim, J. 1993. The non-reductivist's troubles with mental causation. In Heil and Mele 1993, pp. 189-210. Reprinted in Kim 1993a, pp. 336-57.

Lewis, D. 1991. Parts of Classes. Oxford: Basil Blackwell.

Lodder, A. R. 2013. Dutch Supreme Court 2012: virtual theft ruling a one-off or first in a series? Journal For Virtual Worlds Research [S.l.] 6(3): 1-12.

Merricks, T. 2001. Objects and Persons. Oxford University Press.

Noveck, B.; and Balkin, J. 2006. The State of Play: Law, Games, and Virtual Worlds. New York University Press.

Nozick, R. 1974. Anarchy, State and Utopia. Basic Books.

Putnam, H. 1981. Reason, Truth, and History. Cambridge University Press.

Reichenbach, H. 1958. The Philosophy of Space and Time. New York: Dover.

Salmon, Wesley C. 1998. Causality and Explanation. Oxford University Press.

Schiffer, S. 1987. Remnants of Meaning. Cambridge, MA: MIT Press.

Wall, D. 2009. Crime and Deviance in Cyberspace. Routledge.

Walton, K. L. 1990. Mimesis as Make-Believe: On the Foundations of the Representational Arts. Harvard University Press.

Walton, K. 2013. Fictionality and imagination reconsidered. In Fictionalism to Realism: Fictional and Other Social Entities, ed. by C. Babero, M. Ferraris and A. Voltolini. Cambridge Scholars Publishing, pp. 9-26.

Wildman, N. (ms). Learning by fictionally doing. Unpublished manuscript.

Wildman, N. and Woodward, R. 2018. Interactivity, fictionality, and incompleteness. In The Aesthetics of Video Games, ed. by J. Robson and G. Tavinor. Routledge, pp. 112-27.

Woodward, R. 2014. Walton on fictionality. Philosophy Compass 9 (12): 825-36.

Wright, C. 1986. Realism, Meaning and Truth. Second edition. Oxford: Blackwell, 1993.

Zhong, L. 2014. Sophisticated exclusion and sophisticated causation. Journal of Philosophy 111(7): 341-60. 